\title{
Metastasiertes HER2-positives Mammakarzinom
}

\author{
Der Ausschuss für Humanmedizin \\ (CHMP) der Europäischen Arznei- \\ mittel-Agentur (EMA) hat sich für \\ die Zulassung von Pertuzumab \\ ausgesprochen.
}

Rationale für diese Entscheidung sind die Ergebnisse der Zulassungsstudie CLEOPATRA. Bei zuvor gegen die metastasierte Erkrankung nicht vorbehandelten Patientinnen mit HER2-positivem Mammakarzinom reduzierte die zusätzliche Gabe von Pertuzumab zur Kombination
Trastuzumab plus Docetaxel das Sterberisiko signifikant um $34 \%$ (HR 0,66; $p=$ 0,0008). Zum Zeitpunkt der Analyse war das mediane Gesamtüberleben (OS) im Pertuzumab-Arm noch nicht erreicht im Kontrollarm lag es bei 37,6 Monaten. Zusätzlich verlängerte das Pertuzumabbasierte Regime das progressionsfreie Überleben (PFS) von median 12,4 auf 18,5 Monate (HR 0,62; $\mathrm{p}<0,0001)$. Die objektive Ansprechrate (ORR) verbesserte sich unter Pertuzumab von 69,3 auf $80,2 \%(\mathrm{p}=0,0011)$ [Swain $\mathrm{S}$ et al. SABCS
2012;Poster P5-18-26 sowie Baselga J et al. N Engl J Med. 2012;366(2):109-19].

Pertuzumab zeichnet sich durch ein günstiges Sicherheitsprofil aus: Die Abbruchraten aufgrund von unerwünschten Ereignissen waren unter dem Pertuzumab-Regime und der aktuellen Standardtherapie nahezu identisch (6,1 vs. $5,3 \%)$. Die Zulassung von Pertuzumab in der Europäischen Union wird für Februar 2013 erwartet.

red

Nach Informationen von Roche

\section{Die Therapieziele sind ambitionierter geworden}

\author{
Durch die Einführung von Tyrosin- \\ kinasehemmern (TKI) der zweiten \\ Generation haben sich die Be- \\ handlungsoptionen für Patienten \\ mit chronischer myeloischer \\ Leukämie (CML) verbessert.
}

In der Phase-III-Studie ENESTnd (Evaluating Nilotinib Efficacy and Safety in Clinical Trials of Newly Diagnosed PH+ CML Patients) konnte nachgewiesen werden, dass Nilotinib (Tasigna ${ }^{\circ}$ ) bei Patienten mit neu diagnostizierter CML eine wirksame und sichere Therapie ermöglicht: Unter dem Zweitgenerations-TKI wurde ein tieferes und schnelleres molekulares Ansprechen als unter Imatinib beobachtet ( $\mathrm{p}<0,001)$. „Darüber hinaus bot Nilotinib im Vergleich zu Imatinib einen signifikant besseren Schutz vor einer Progression der Erkrankung in die akzelerierte Phase oder Blastenkrise ( $\mathrm{p}=0,01$, bzw. $\mathrm{p}=0,004$ für die Dosierungen $300 \mathrm{mg}$ 2-mal täglich und $400 \mathrm{mg}$ 2-mal täglich), und es kam zu weniger CML-bedingten Todesfällen", führte Andreas Hochhaus, Jena, aus [Saglio G et al. N Engl J Med. 2010;362(24):2251-2. Hochhaus A et al. EHA Meeting 2011;Abstract 0484].

Der bereits früh nachweisbare, signifikante Unterschied in den molekularen Ansprechparametern zugunsten von $\mathrm{Ni}$ lotinib konnte auch nach drei Jahren bestätigt werden $(\mathrm{p}<0,0001$ [Larson RA et al. Leukemia. 2012;26(10):2197-203]). Seit der Erhebung der 2-Jahres-Daten wurde bei keinem weiteren Patienten eine Progression beobachtet. Mit fünf im Vergleich zu 14 CML-bedingten Todesfällen wurde unter 2-mal täglich $300 \mathrm{mg}$ Nilotinib außerdem im Vergleich zu 1-mal täg-

lich $400 \mathrm{mg}$ Imatinib eine signifikant höhere Gesamtüberlebensrate erreicht ( $\mathrm{p}=$ 0,0356).

Silke Wedekind

Satellitensymposium „Innovationen eröffnen

Perspektiven“ im Rahmen der DGHO-Jahrestagung, Stuttgart, 21.10.2012; Veranstalter: Novartis Pharma

\section{Nebenwirkungsmanagement bei Cabazitaxel-Therapie \\ Febrile Neutropenie im Blick}

\section{Moderne Chemotherapien beim metastasierten kastrationsresisten- ten Prostatakarzinom (mCRPC) erfordern mehr Aufmerksamkeit für die Neutropenie.}

Patienten mit mCRPC, bei denen die Erkrankung trotz Docetaxel-Behandlung fortschreitet, profitieren von einer weiteren Behandlung mit Cabazitaxel [de Bono et al. Lancet. 2010;376(9747):1147-54]. Allerdings entwickelten fast $8 \%$ der Patienten unter Cabazitaxel in der TROPIC-Studie eine febrile Neutropenie (FN) dritten oder vierten Grades. Wie gefährlich die FN sein kann, belegt eine weitere Studie: Demnach stirbt einer von zehn Patienten, der wegen einer FN ins Krankenhaus aufgenommen wird - trotz entsprechender stationärer Therapie. Komorbiditäten steigern die Mortalitätsrate deutlich, sodass bei einer zusätzlichen Komorbidität eine Mortalität von $20 \%$ auftritt [Kuderer NM et al. Cancer. 2006;106(10): 2258-66]. Die FN kann im praktischen Alltag durch den prophylaktischen Einsatz von Granulozyten-koloniestimulierenden Wachstumsfaktoren (G-CSF) verhindert werden.

Besonders bewährt zur Primärprophylaxe hat sich nach Meinung von Joachim Kleeberg, Stuttgart, der nur einmal pro Zyklus zu verabreichende pegylierte G-CSF Pegfilgrastim (Neulasta ${ }^{\circledR}$ ). Die Einmalgabe pro Chemotherapiezyklus bietet besonders den ambulant behandelten Prostatakarzinompatienten Vorteile. red

Nach Informationen von Amgen 